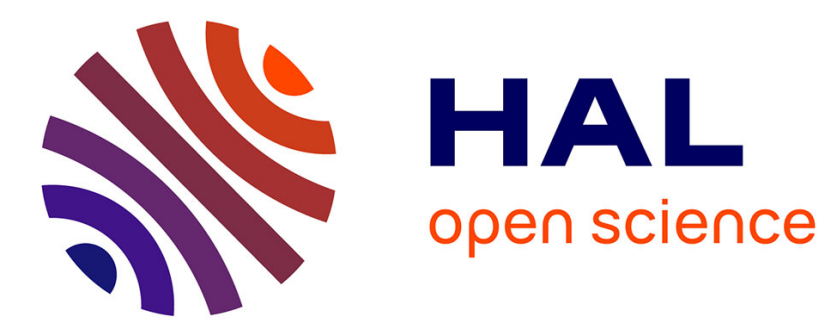

\title{
Expertise économique et gestion publique des problèmes environnementaux
}

\author{
Yannick Rumpala
}

\section{To cite this version:}

Yannick Rumpala. Expertise économique et gestion publique des problèmes environnementaux: Mobilisation et utilisation d'un savoir particulier dans un champ de l'univers politico-administratif français. L'Année de la Régulation, 2004, 8, pp.ISBN 2-7246-0951-4. hal-00351196

\section{HAL Id: hal-00351196 https://hal.science/hal-00351196}

Submitted on 8 Jan 2009

HAL is a multi-disciplinary open access archive for the deposit and dissemination of scientific research documents, whether they are published or not. The documents may come from teaching and research institutions in France or abroad, or from public or private research centers.
L'archive ouverte pluridisciplinaire HAL, est destinée au dépôt et à la diffusion de documents scientifiques de niveau recherche, publiés ou non, émanant des établissements d'enseignement et de recherche français ou étrangers, des laboratoires publics ou privés. 


\title{
Expertise économique et gestion publique des problèmes environnementaux. \\ Mobilisation et utilisation d'un savoir particulier dans un champ de l'univers politico-administratif français
}

\author{
Yannick RUMPALA ${ }^{1}$
}

Résumé. L'intervention publique en matière d'environnement a connu des développements dont une part de plus en plus notable a pris appui sur des courants de réflexion empruntés au champ de la science économique. Cet article revient en particulier sur les processus et logiques par lesquels des liens se sont formés et renforcés, notamment depuis le début des années 1990, entre l'activité politicoadministrative traitant d'enjeux environnementaux et les travaux en économie de l'environnement. L'analyse cherche ainsi les raisons pouvant expliquer l'intérêt croissant que les acteurs publics concernés ont trouvé dans cette branche disciplinaire. Cette dynamique témoigne d'une circulation d'idées qui dépasse amplement le cadre national. En revenant sur les voies de l'institutionnalisation des relations entre milieux bureaucratiques et scientifiques, l'analyse vise à éclairer conjointement les effets perceptibles sur les orientations décisionnelles. Il s'agit de mieux saisir non seulement les situations dans lesquelles ont pu prendre forme des enjeux d'expertise, mais aussi la gamme de débouchés qui ont pu les prolonger. L'examen des attentes à l'égard des travaux économiques et le retour sur les utilisations faites à partir de ces savoirs scientifiques particuliers permettent de préciser cet éclairage et mettent au surplus en évidence les inflexions qui peuvent toucher les modalités de prise en charge publique des questions touchant à la nature.

\footnotetext{
1. $\Xi$ Université de Nice/Faculté de droit, Équipe de Recherche sur les Mutations de l'Europe et de ses Sociétés (ERmes), Avenue Doyen Louis Trotabas, 06050 Nice, Cedex 01 - tél. : 0493866092 - rumpala@unice.fr
} 
À quoi voit-on qu'une question devient sérieuse ? Peut-être au fait qu'elle soit saisie et investie par l'expertise économique. C'est apparemment le cas pour les questions d'environnement: ces dernières semblent être devenues une affaire suffisamment sérieuse pour ne plus devoir être réservée aux seuls écologistes. De fait, les enjeux correspondants, au-delà des hésitations encore perceptibles, bénéficient d'une certaine reconnaissance dans les sphères institutionnelles occupées à essayer de gérer la marche de la société. Autour du vaste thème de l'environnement, un champ d'intervention, aux contours certes encore flous, s'est structuré et inséré dans le système politico-administratif. Dans le champ scientifique, diverses disciplines ont élargi ou renforcé leurs investigations dans ce domaine.

Ces différents développements ne se sont pas produits de manière autonome : ils laissent aussi voir des interactions de plus en plus denses entre les différents champs intéressés. Face aux problèmes environnementaux rendus visibles, le travail collectif qui sous-tend la régulation publique s'est ainsi solidifié en cherchant à prendre appui sur un ensemble de connaissances fiables. Dans cette dynamique, la science économique et son application aux enjeux environnementaux tendent à se voir conférer une position stratégique, et les milieux décisionnels publics semblent effectivement accorder une importance accrue à la recherche et à la production de connaissances dans ce domaine.

Cet article vise donc à aborder ce domaine d'intervention publique par le biais des «technologies de gouvernement» qui y sont déployées (Miller, Rose [1990]). Plus précisément, son objectif est de saisir dans quelle mesure et comment les travaux en économie de l'environnement, champ disciplinaire luimême en plein essor, sont mis à contribution dans la sphère politicoadministrative. Dans quelles conditions le savoir économique est-il mobilisé ? Qui sont les utilisateurs des lumières proposées par les économistes ? Quelles sont leurs motivations? Que font-ils des analyses et outils qui sont à leur disposition? Plus largement, si la réflexion économique gagne une influence croissante dans l'activité politico-administrative concernant l'environnement, il s'agit de pénétrer les processus par lesquels cette progression peut avoir lieu et de mettre en relief les effets qui peuvent se faire sentir dans les orientations publiques. Un tel questionnement tend à amener l'analyse dans le sillage d'hypothèses qui ont, depuis quelques décennies, été déjà largement explorées sur d'autres aspects. En l'occurrence, l'hypothèse centrale serait de placer les processus repérables dans le cadre d'un mouvement plus général de «scientifisation de la politique $^{2} »$. La prise en charge institutionnelle des problèmes environnementaux laisse toutefois entrevoir des tendances qui amènent à préciser une telle hypothèse : le recours à l'économie s'insérerait notamment dans une logique de rationalisation gestionnaire elle-même en développement ${ }^{3}$. L'installation de

2. Cette hypothèse prolongerait notamment les analyses de Jürgen Habermas [1973]. Dans ce même prolongement et dans une perspective davantage centrée sur la science économique, voir Jon D. Wisman [1990].

3. Cette dynamique viendrait alors constituer une composante du processus de «tutélarisation écouménique » dont nous avons exposé les ressorts dans Rumpala [2003]. 
L'installation de raisonnements fondés sur des cadres et des références économiques est alors un phénomène qui est loin d'être anodin, dans la mesure où cela peut produire des effets de cadrage dans la manière d'appréhender les problèmes et dans la recherche de voies d'action.

Saisir ce qui s'apparente à une interface requiert d'ailleurs des outils d'analyse appropriés, c'est-à-dire capables de saisir conjointement les logiques d'action confrontées dans la rencontre de ces différents champs. Nous combinerons pour cela les apports de la sociologie de l'action publique et ceux de la sociologie des sciences. Pour notre investigation, il faut en effet, d'une part, appréhender non seulement le travail intellectuel qui sous-tend les stratégies des acteurs publics ${ }^{4}$, mais aussi les facteurs dont dépend la réception des arguments dans l'espace de discussion politico-administratif5. D'autre part, il s'agit de pouvoir suivre des idées en circulation et, plus précisément les dynamiques qui permettent d'expliquer les ralliements autour de certaines des conceptions avancées. Les travaux développés dans le cadre du Centre de sociologie de l'innovation, notamment par Michel Callon [1988] et Bruno Latour [1995], offrent pour cela une base pertinente.

Trois axes seront ainsi successivement suivis pour pénétrer les interactions tissées, à partir des enjeux environnementaux, entre le travail politicoadministratif et la réflexion économique. Le premier cherchera les raisons pouvant expliquer l'intérêt croissant que les acteurs publics concernés ont trouvé dans l'économie de l'environnement. Le deuxième, profitant de la nouveauté relative des problématiques environnementales, reviendra sur les voies de l'institutionnalisation des relations entre milieux bureaucratiques et scientifiques. Cela permettra d'en éclairer les effets sur les dynamiques décisionnelles, et notamment de souligner les orientations ainsi amenées dans la prise en charge publique des questions touchant à la nature. Le troisième examinera les utilisations qui sont faites de la réflexion économique, en gardant d'ailleurs une attention pour les adaptations et déplacements qui peuvent s'ensuivre dans les logiques d'action étatiques.

\section{LES RAISONS D'UNE SÉDUCTION}

La prise en charge publique des enjeux environnementaux se construit en passant par un espace de discussion marqué par la concurrence entre discours et argumentaires. Si les propositions élaborées en économie de l'environnement trouvent une audience dans la sphère politico-administrative, il faut d'abord

4. En s’intéressant au rapport entre idées et recettes de politiques publiques, la réflexion engagée par Bruno Jobert ouvre des perspectives utiles, dans la mesure où elle met en évidence le rôle et l'influence des «forums professionnels d'experts », notamment des «forums scientifiques des économistes », dans la construction des politiques publiques et de leurs argumentaires justificatifs (cf. Bruno Jobert [1993], et «Introduction : Le retour du politique », dans Bruno Jobert (dir.) [1994]).

5. Le travail de Giandomenico Majone [1989] fournit des compléments précieux. 
préciser les facteurs et conditions qui permettent cette réception et qui assurent une forme de reconnaissance à ce savoir spécialisé.

\section{Un développement de travaux qui peut nourrir l'action publique}

À l'exception de travaux antérieurs épars, les développements qui vont fonder l'économie de l'environnement en tant que branche disciplinaire remontent principalement aux réflexions engagées au milieu des années $1960^{6}$. Ces développements deviennent notables en France dans les années 19707, mais c'est surtout dans les années 1980 que les démarches de structuration se font plus affirmées ${ }^{8}$. La production scientifique - rapports, livres, publications sous forme d'articles, numéros spéciaux de revues académiques... (Godard [1998, p. 28-29]) - augmente et trouve des possibilités de circulation (colloques, conférences...). Malgré un nombre de chercheurs relativement restreint et une dispersion apparente dans les équipes de recherche (Godard [1998, p. 36]), une base intellectuelle a ainsi pu progressivement se constituer, et ce en donnant aussi à voir des possibilités d'application dans le registre pratique.

Certes, le champ des économistes travaillant dans cette branche disciplinaire n'est pas uniforme. Il y a en effet une grande diversité dans les démarches, les écoles de pensée, rattachables à l'économie de l'environnement. Toutefois, les chercheurs et universitaires spécialisés dans ce domaine tendent à former une communauté, et plus précisément ce que Peter M. Haas [1992] appelle une «communauté épistémique ». C'est-à-dire un ensemble de professionnels disposant d'une compétence reconnue dans un domaine particulier, partageant une série de convictions sur les caractéristiques des questions en jeu, sur les explications causales à privilégier, sur les principes d'action à promouvoir, et jouissant d'une autorité scientifique suffisante pour pouvoir prétendre à l'emploi des connaissances produites dans un champ d'intervention publique.

Le travail des économistes sur les questions d'environnement s'est renforcé au point de paraître capable de fournir aux décideurs un équipement analytique et conceptuel, transposable et utilisable pour que ces derniers puissent étayer leurs réflexions. Autrement dit, ce travail parait doté de qualités suffisantes pour pouvoir participer aux processus décisionnels et guider les choix à faire. C'est ce type de sentiment que va par exemple exprimer Jacques Theys, Directeur scientifique de l'IFEN (Institut français de l'environnement) et Chef du Centre de prospective et de veille scientifique des ministères de l'Environnement et de

6. Trois séries de réflexions reviennent couramment comme références : celles de Kenneth Boulding [1966], celles de John Krutilla [1967] et celles de Robert Ayres et Allan Kneese [1969].

7. La création du CIRED (Centre international de recherche sur l'environnement et le développement), sous l'égide d'Ignacy Sachs, date de 1973. Celle du Département d'Économie de l'Environnement du Centre Économie-Espace-Environnement (C3E) remonte à 1978. Son fondateur, le professeur René Passet, publiera l'année suivante un ouvrage remarqué [1979].

8. Pour des précisions historiques sur le développement des recherches en économie de l'environnement en France, voir Olivier Godard [1998]. 
l'Équipement, dans le cadre du colloque Environnement économie de février 1993: «La troisième bonne nouvelle que nous avons apprise hier et aujourd'hui, est que des progrès sensibles ont été faits depuis dix ans en matière de connaissance et que désormais l'économie de l'environnement a atteint un niveau de maturité suffisant pour fournir les outils théoriques ou appliqués qu'exige le passage à cette nouvelle étape de l'intégration $[. . .]^{9}$.»

\section{La scientificité de l'économie et ses attraits}

Cette attirance pour le savoir proposé par l'économie de l'environnement opère en fait à deux niveaux. D’une part, les débouchés de cette branche disciplinaire paraissent plus fructueux, et les connaissances et outils qui en sont issus gagnent ainsi en crédibilité. D'autre part, l'économie elle-même figure souvent dans les esprits comme un corps de connaissances semblant offrir des garanties de scientificité. Grâce notamment à l'appareillage mathématique qui la soutient en partie, l'économie tend à être couramment considérée comme la plus dure des sciences molles ${ }^{10}$. Cette opinion imprègne de nombreux discours politiques et administratifs, et elle peut effectivement être retrouvée dans les travaux institutionnels concernant l'environnement. Elle apparait par exemple dans les travaux réalisés dans le cadre du Commissariat général du Plan pour le XIe Plan, sous la modalité suivante : «[...] malgré sa relativité, l'économie reste néanmoins la plus scientifique des sciences sociales: elle a ses méthodes qui reposent sur les chiffres, les observations, les évaluations, les comparaisons » (Commissariat général du Plan [1993, p. 16].)

Au surplus, l'attention croissante dont bénéficient les analyses économiques est liée à l'appréhension de la réalité que les économistes ont été capables d'installer. Comme l'ont rappelé Michel Callon et Bruno Latour [1997, p. 47-49], le travail de la science économique est aussi un travail performatif : l'économie en tant que discipline scientifique façonne l'objet qu'elle prétend décrire, c'est-àdire l'économie en tant qu'ensemble d'activité ${ }^{11}$. C'est pourquoi il est important de bien identifier les cadres théoriques et catégories utilisés par les économistes lorsqu'ils s'intéressent aux questions d'environnement.

9. Theys ([1993], p. 40). La partie en italique est celle du texte original. Les deux premières nouvelles concernaient respectivement «la prise de conscience de l'importance des enjeux économiques liés à l'environnement» et les changements dans «la perception des rapports économie-environnement».

10. Sur les tensions résultant de cette quête de scientificité, voir Frédéric Lordon [1997].

11. cf. «Introduction : the embeddedness of economic markets in economics », dans Michel Callon (ed.) [1998]. 


\section{L’influence des orientations théoriques néoclassiques}

L'appréhension de ces questions dans la discipline économique s'inscrit pour une large part dans le prolongement des approches néoclassiques ${ }^{12}$. C'est cette influence qui non seulement imprègne les réflexions des milieux académiques, mais qui se fait aussi sentir jusque dans les institutions publiques travaillant dans ce domaine. Le cadre théorique néoclassique y est en position centrale et sert communément de fondation à l'ambition scientifique des analyses économiques. Dans ce cadre théorique attaché au bon fonctionnement des «mécanismes du marché ", les ressources naturelles tendent à être réduites à un capital, à un ensemble d'inputs pour lesquels la préoccupation environnementale se résume le plus souvent à déterminer la manière la plus rationnelle de les utili$\operatorname{ser}^{13}$.

Le caractère normatif de la théorie néoclassique a été maintes fois souligné : elle ne dit pas ce qui est, mais ce qui doit être (Guerrien [1996a], et de manière plus rapide Guerrien [1996b, p. 352-355]). Les économistes qui suivent de près ou de loin la voie néoclassique conservent généralement une vision du monde dans laquelle la raréfaction de ressources naturelles peut être maitrisée par une gestion efficace, assimilable à un problème d'optimisation. De la sorte, il n'y aurait pas de raison à ce que les logiques de développement censées fonder la prospérité économique entrent fatalement en opposition avec la préservation des équilibres écologiques et des milieux naturels.

Les postulats néoclassiques ont toutefois suscité des réactions qui ont amené à questionner plus profondément les conditions de cette compatibilité. Sous l'étiquette ecological economics s'est ainsi développé un courant réclamant une approche plus interdisciplinaire pour appréhender de manière adéquate l'enchâssement des activités économiques dans l'écosphère ${ }^{14}$. Ce courant a su développer le type d'attributs propres à la profession, attributs qui lui ont permis de renforcer sa structuration et de gagner une respectabilité académique. Depuis septembre 1987, l'International Society for Ecological Economics a rallié un nombre non négligeable d'économistes à l'échelle internationale. Cette association savante dispose également, depuis 1989, de sa propre revue: Ecological Economics. Une branche européenne (European Society for Ecological Economics) a été créée en 1996, avec à sa présidence Sylvie Faucheux, professeur de science économique à l'Université de Versailles/Saint-Quentin-en-Yvelines. L'ESEE a fait un gros effort pour être reconnue au niveau européen, notamment à la DG XII (DG Recherche). Toutefois, dans les milieux administratifs intéressés, ce

12. Pour une présentation synthétique, voir par exemple Franck-Dominique Vivien [1994, p. 45 et sq.]. Pour un exposé plus critique, voir Michael Jacobs [1994].

13. Comme dans cet extrait de la synthèse du rapport «Croissance et environnement » préparée par la direction de la Prévision du ministère de l'Économie et des Finances : «L'approche économique des problèmes d'environnement recouvre une grande variété de concepts. On peut la résumer en disant que l'environnement est une ressource ou un bien que les mécanismes de marché ne conduisent pas à exploiter ou allouer de façon efficace. » (Ministère de l'Économie et des Finances [1992, p. 5] ; la partie en italique est celle du texte original.)

14. Pour une appréhension historique et épistémologique de ce courant, voir Clive L. Spash [1999]. 
(DG Recherche). Toutefois, dans les milieux administratifs intéressés, ce courant ne parait pas encore suffisamment opérationnel pour pouvoir être utilisé largement.

Une conceptualisation qui joue un rôle prégnant dans l'appréhension des problèmes : les effets externes

Les catégories économiques qui circulent dans l'espace de discussion témoignent cependant de l'influence prépondérante que conservent la perspective néoclassique et son appareillage conceptuel. La problématisation installée dans cette perspective donne en effet un rôle de pivot à la notion d'effet externe (externalité est un autre terme utilisé) ${ }^{15}$ dans les analyses concernant l'environnement ${ }^{16}$. L'absence de prise en considération des effets externes environnementaux tend à être vue comme un obstacle sur le chemin de l'efficacité. Lorsque le domaine de l'environnement est abordé sous un angle économique, le discours institutionnel tend à reprendre le même type d'énoncés de base que celui qui est admis par la littérature néoclassique. De manière caractéristique, la synthèse du rapport «Croissance et environnement » préparée par la direction de la Prévision du ministère de l'Économie et des Finances aligne des formules ordinaires dans ce type de littérature, affirmant par exemple : «Les problèmes de l'environnement s'interprètent principalement en termes d'effets dits 'externes' » (Ministère de l'Économie et des Finances [1992, p. 5].)

Une telle approche conduit à de nombreux efforts de calcul pour évaluer ces effets externes. Par l'intermédiaire de son budget de recherche, l'Agence de l'environnement et de la maittrise de l'énergie (ADEME) est une de ces institutions étatiques qui soutiennent ce type d'efforts pour analyser en termes économiques les impacts environnementaux de divers secteurs (industries, filières énergétiques, transports...). L’intention qui vient généralement en prolongement est de pouvoir « internaliser » ces effets externes. Mais il reste une importante série de problèmes théoriques et pratiques, car les tentatives de chiffrage sous forme monétaire s'attaquent à des facteurs qui ont justement pour particularité de sortir du cadre marchand traditionnel.

15. Et aussi à celle de « biens collectifs».

16. Pour une présentation de la notion et de ses limites, voir Franck-Dominique Vivien [1994, p. 48-51 et 90-95]. Sans s'engager dans des développements qui sortiraient du cadre de notre investigation, précisons cependant que, dans l'analyse économique, ce concept d'externalité sert couramment à désigner tout effet indirect résultant d'activités de production ou de consommation d'un ou de plusieurs agents économiques, et touchant le bien-être d'autres agents. Il s'agit d'une situation où il n'y a pas d'échange ou de transaction entre ces agents, donc pas de passage par un système de prix, et l'effet n'est pas pris en compte dans les décisions du ou des agents qui engendre(nt) cette situation. Dans le cas de l'environnement, les préoccupations portent principalement sur les externalités négatives, celles provenant des pollutions et nuisances. 


\section{L'influence des travaux de l'OCDE}

Les références et analyses qui circulent dans l'espace de discussion institutionnel montrent par ailleurs que les travaux de l'OCDE (Organisation de coopération et de développement économiques) exercent une influence importante dans les débats. De ces travaux ont émergé des problématisations qui ont contribué à orienter profondément les interventions publiques dans le domaine de l'environnement.

Dans le champ des relations internationales, l'OCDE tend à se placer comme un pôle d'expertise économique pour les pays industrialisés. Le positionnement de cet organisme est particulier, car ses travaux abordent essentiellement les questions d'environnement avec une approche centrée sur les aspects économiques. À cet égard, il faut signaler le rôle central que joue la division de l'Économie au sein de la direction de l'Environnement de l'OCDE. Cette composante a non seulement largement encouragé les études effectuées par des économistes, mais s'est aussi efforcée de les diffuser sous diverses formes de publications. Des manuels, des guides pratiques ont été édités pour «éclairer» les décideurs et les aider dans leurs tâches. C'est aussi par ce type d'intermédiaires, de dispositifs d'intéressement ${ }^{17}$, qu'ont pu être poussées des techniques économiques destinées à évaluer des projets ou des politiques ${ }^{18}$.

Les groupes de travail que l'OCDE organise et auxquels sont conviés des représentants des administrations des pays membres constituent un autre lieu pour l'acclimatation des idées propres aux approches économiques. C'est à ce répertoire d'idées que peut être rattachée une part des recommandations faites à la France par le groupe de l'OCDE sur les performances environnementales: dans une logique de renforcement de l'efficacité des interventions, le rapport d'évaluation souligne l'importance de la disponibilité d'informations, surtout pour pouvoir asseoir les politiques sur des analyses coûts/avantages ou, à défaut, sur des analyses coûts/efficacité (OCDE [1997]).

Somme toute, ces différents éléments, qui renvoient aux avancées de la branche disciplinaire, mais aussi aux soutiens extérieurs qu'elle peut trouver, contribuent à donner une crédibilité aux travaux en économie de l'environnement. Ces travaux offrent ainsi des ressources autour desquelles peut s'effectuer une agrégation d'intérêts. Des alliances vont pouvoir prendre forme, et surtout s'insérer dans le tissu institutionnel.

17. Nous reprenons avec cette conceptualisation le cadre d'analyse de Michel Callon et Bruno Latour. Cf. sur le «modèle de l'intéressement ", Madeleine Akrich, Michel Callon, Bruno Latour [1988], notamment p. 15-17. Le verbe intéresser est ici à appréhender dans le sens de faire prendre intérêt à quelque chose.

18. L'argumentation avancée tend même à faire du recours aux économistes un point de passage obligé pour parvenir à véritablement préserver l'environnement : «L'économie est un facteur important pour les personnes prenant des décisions ayant un effet sur notre environnement. L'environnement continuera de pâtir si les économistes ne jouent pas un plus grand rôle dans l'analyse et l'élaboration de la politique. Tel est le message principal de ce manuel. » (OCDE [1995, p. 11].) 


\section{UN PROCESSUS D’INSTITUTIONALISATION}

La partie précédente visait à donner des éléments d'explication sur l'attirance que peut exercer l'économie de l'environnement sur des responsables politiques ou administratifs, autrement dit à cerner les facteurs qui peuvent participer à l'intéressement de ces acteurs. Il faut maintenant préciser comment des liens ont pu concrètement se mettre en place entre les champs d'activités. Comprendre les stratégies déployées suppose de prolonger l'investigation au-delà des ensembles argumentatifs élaborés, dans la mesure où leur circulation ne peut être comprise sans restituer l'espace de discussion commun dans lequel ils entrent. Précisément, il s'agit de porter l'attention sur la structuration de réseaux et sur la dynamique d'institutionnalisation qui est ainsi rendue possible ${ }^{19}$.

Un élargissement des possibilités de participation institutionnelle pour les économistes : l'imbrication tendancielle des epistemic communities dans les policy communities

L'intérêt des milieux publics pour ces réflexions économiques peut d'abord être relié aux caractéristiques du personnel travaillant sur les questions d'environnement dans la sphère administrative et dans son orbite. Comme l'a fait remarquer Corinne Larrue [1995, p. 45], les fonctionnaires de l'administration de l'environnement sont généralement des ingénieurs ou des juristes, donc peu familiers des mécanismes économiques. L'évolution perceptible à travers la montée des approches économiques dans les politiques environnementales peut ainsi s'expliquer par l'entrée dans ce domaine administratif de personnes aux profils plus proches de celui des économistes. Il s'agit notamment de personnes formées à l'École nationale de la statistique et de l'administration économique (ENSAE) et/ou passées par la direction de la Prévision du ministère de l'Économie et des Finances.

Au surplus, l'insertion des économistes apparait de plus en plus courante dans les groupes de réflexion institutionnels constitués sur les thèmes environnementaux. Leur contribution est même d'autant plus recherchée lorsque les enjeux environnementaux croisent des enjeux économiques. Les travaux engagés dans le cadre de la commission «Environnement, qualité de vie, croissance » du XIe Plan illustrent bien cette tendance. L'exemple le plus marquant est celui de l'Atelier 1 («Environnement, économie et croissance»), qui était chargé d'étudier les outils d'intervention publique pour analyser les liens entre la croissance et l'environnement, et dont la plupart des membres peuvent être rattachés au milieu des économistes.

19. L'utilisation que nous faisons de cette notion de réseau reste essentiellement dans le prolongement du cadre d'analyse de Michel Callon et Bruno Latour. Comme l'explique par exemple ce dernier: «[...] chaque fois qu'il est question d'une application réussie d'une science, il faut rechercher l'extension progressive d'un réseau. » (Latour [1995, p. 602].) 
À un autre niveau, signalons également des rapprochements qui ont pu s'établir au sein des partis politiques du courant écologiste. En faisant partie du bureau national de Génération écologie, Lionel Stoléru, qui a enseigné l'économie pendant une partie de sa carrière ${ }^{20}$, était en position de faire passer certaines conceptions dans le parti fondé par Brice Lalonde. Alain Lipietz, X-Ponts, directeur de recherche au CNRS, économiste au Centre d'études prospectives d'économie et mathématiques appliquées à la planification (CEPREMAP) ${ }^{21}$, va se trouver dans une position similaire chez Les Verts, en devenant porte-parole de la Commission économique et ainsi un des principaux inspirateurs du programme économique du parti (Lipietz [1992]).

L'insertion et le développement de services ad hoc dans les structures administratives

La création et le développement de services à vocation économique a pris également un rôle important dans l'implantation de ce type de perspective au sein des structures administratives chargées de l'environnement. Au ministère de l'Environnement, le regard propre à la discipline économique va être confirmé et intégré parmi les missions du Service de la recherche et des affaires économiques (SRAE)22. En l'occurrence, ce service devait " promouvoir le développement, en France, de l'économie de l'environnement et, en liaison avec les directions concernées du ministère, des mesures économiques favorisant des modalités de croissance plus respectueuse de l'environnement ${ }^{23} »$. Parmi les quatre bureaux du SRAE, c'est celui des affaires économiques et sciences humaines qui était plus particulièrement chargé d'« animer la réflexion économique collective du ministère et [d'] augmenter les capacités globales d'évaluation et d'expertise économiques» (Ministère de l'Environnement/SRAE [1994, p. 6]).

La dynamique s'est avérée similaire dans les établissements sous tutelle. De son côté, l'ADEME a en effet mis en place un service «Économie et Prospective ». L'Institut national de l'environnement industriel et des risques (INERIS) a également constitué un groupe «Évaluation et études économiques».

Au ministère de l'Environnement, ce mouvement en faveur des approches économiques a été plus récemment renforcé avec la création d'une cinquième direction d'administration centrale, baptisée Direction des études économiques

20. De 1981 à 1988 à l'École polytechnique, après 1992 à l'École des Mines de Paris. Lionel Stoléru a passé un $\mathrm{Ph}$.-D en sciences économiques à l'Université de Stanford en Californie avec le professeur Kenneth J. Arrow.

21. Voir la notice qui lui est consacrée dans Jacques Julliard et Michel Winock (dir.) [1996, p. 721-722].

22. Le SRAE succède au SRETIE (Service de la recherche, des études et du traitement de l'information sur l'environnement), lequel comportait une Mission des études économiques, des statistiques et du plan.

23. Arrêté du 11 janvier 1994 portant organisation de directions de l'administration centrale du ministère de l'Environnement. 
et de l'évaluation environnementale (D4E). Cette nouvelle direction a été placée en mai 2000 sous la responsabilité de Dominique Bureau, polytechnicien, ingénieur en chef des Ponts et Chaussées, et auparavant membre du Conseil d'analyse économique du Premier ministre ${ }^{24}$. Cinq nouveaux bureaux sont créés, dont un spécifiquement consacré à l'économie de l'environnement.

Cette innovation organisationnelle s'insère dans un courant de réflexions sur la capacité de réponse du ministère de l'Environnement face à des thématiques ayant une forte dimension économique. Le rapport d'Edmond Malinvaud [1997], figure respectée de la science économique française, avait déjà souligné une série de lacunes plus générales. Les recommandations avancées ont permis de motiver le lancement d'une mission au début 1998 afin que celles-ci puissent être approfondies. Pour cela, le directeur général de l'Administration et du Développement du ministère de l'Environnement, Jean-Luc Laurent, a fait une demande d'appui au directeur général de l'INSEE, Paul Champsaur, et au conseil général des Mines ${ }^{25}$. La mission a ainsi été confiée à Pascal Mazodier, inspecteur général de l'INSEE, Henri Prévot, Ingénieur général des mines, et Sylviane Gastaldo, Directeur des études de l'ENSAE, qui ont remis leur rapport en octobre 1998. Les analyses présentées sont venues constituer un réservoir d'arguments supplémentaires pour justifier un repositionnement institutionnel du ministère, repositionnement que devait en l'occurrence matérialiser la nouvelle D4E.

Il faut d'ailleurs signaler que la création de cette direction répond aussi pour une part importante à la recherche de ressources légitimatoires. La rencontre d'enjeux environnementaux et d'enjeux économiques a en effet de plus en plus souvent nourri une zone de controverses, dans laquelle la maitrise de l'expertise est devenue un avantage capital. Pour le ministère de l'Environnement s'est ainsi posée la question de pouvoir entrer en concurrence avec d'autres ministères mieux armés en termes de compétences économiques et pouvant donc se situer dans une position plus favorable pour d'éventuels arbitrages. C'est un point que le rapport de Sylviane Gastaldo, Pascal Mazodier et Henri Prévot soulignait d'une autre manière en mettant en évidence l'intérêt que ce ministère aurait à se constituer en interlocuteur crédible : «Au total, vis-à-vis des autres ministères, c'est bien sa capacité de contestation économique que doit développer le MATE s'il veut pouvoir contrer efficacement tel ou tel groupe de pres$\operatorname{sion}^{26} . »$

24. Corinne Lepage, ancienne ministre de l'Environnement, a vu dans cette nomination un signe d'assujettissement au ministère de Finances ( $c f$. Corinne Lepage [2000, p. 26-27]). Dominique Bureau avait effectivement travaillé à la direction de la Prévision en tant que chargé de mission au Bureau des transports.

25. Cf. Lettre du 16 janvier 1998 en Annexe A1, dans Sylviane Gastaldo, Pascal Mazodier, Henri Prévot [1998, p. 35]. La demande officialisait une proposition faite par Bernard Guibert (chef du bureau des Affaires économiques et sciences sociales au SRAE), qui avait déjà préparé la contribution du ministère de l'Aménagement du territoire et de l'Environnement à la mission confiée par le Premier Ministre à Edmond Malinvaud en juillet 1996.

26. «Une faible propension à utiliser le langage économique » dans Gastaldo, Mazodier, Pré$\operatorname{vot}([1998$, p. 11]) 
Recours aux économistes comme experts

et mobilisation de travaux hors du champ scientifique

Ces développements organisationnels ont accompagné une extension des démarches de consultation plus ou moins formelles en direction des économistes de l'environnement. Ces derniers ont pu de plus en plus souvent profiter de la mise en forme d'une demande institutionnelle. En s'efforçant d'y répondre, ils s'insèrent ainsi dans des dynamiques d'expertise à différents échelons étatiques. À partir de leurs travaux, certains spécialistes de l'économie de l'environnement tendent précisément à se placer dans une logique dans laquelle il s'agit de contribuer aussi à éclairer les décideurs. La position médiatrice de ces économistes gagne en fait une influence à la mesure des gages scientifiques qu'ils ont été capables de produire.

Les grands noms dans cette branche de l'économie, ceux qui sont dotés d'un capital scientifique éminent, se voient plus fréquemment confier des responsabilités dans les structures et procédures institutionnelles. L'atelier « Environnement, économie, croissance» de la commission «Environnement, qualité de vie, croissance » du XIe Plan avait ainsi pour vice-président Paul Zagamé, professeur à l'Université Paris I, et pour rapporteur général Olivier Godard, alors chargé de recherche au CNRS, au Centre international de recherche sur l'environnement et le développement (CIRED). Paul Zagamé, outre ses fonctions de conseiller scientifique au Commissariat général du Plan, sera par la suite membre de la Commission française du développement durable. Olivier Godard entrera en décembre 1997 dans le comité de pilotage de la cellule «Prospective et stratégie» du ministère de l'Environnement (en remplacement de Dominique Bureau, parti au Conseil d'analyse économique).

Le séminaire commun organisé en 1998-1999 par le Commissariat général du Plan, la direction de la Prévision, et le ministère de l'Environnement, est un autre exemple de ces espaces d'échanges d'idées qui sont aménagés dans une logique proche de l'expertise. Dans un but d'information des responsables concernés et de clarification des débats, ce séminaire a consisté à réunir mensuellement pendant douze séances (plus une exceptionnelle) à partir d'avril 1998 des producteurs et utilisateurs d'études et de recherches en économie de l'environnement. Ce séminaire a débouché sur un colloque Économie de l'environnement, destiné à un plus large public, le mercredi 19 janvier 2000.

Les années 1990 voient, en outre, le développement de démarches plus ou moins coordonnées visant à mobiliser dans le champ administratif les ressources que semblent offrir les travaux de recherche ${ }^{27}$. En 1992, le sénateur Bernard Hugo rend ainsi un rapport d'information sur les aspects économiques des politiques d'environnement. Face aux interrogations subsistant sur les connexions entre environnement et économie, l’idée guidant ce rapport est

27. Les initiatives repérables auparavant apparaissent plus isolées et leurs apports, relativement ponctuels. Voir, par exemple, le travail réalisé par le Groupe interministériel d'évaluation de l'environnement [1975]. 
d'« apporter un peu de clarté dans des débats confus et quelques éléments d'appréciation sur des stratégies possibles » (Hugo [1992, p. 7]). L'intérêt pour une approche économique des problèmes est aussi largement présent dans le rapport « Croissance et environnement » pour lequel plusieurs services ministériels et centres d'études ont apporté leur contribution.

La Commission française du développement durable et le Commissariat général du Plan ont monté en commun, en 1997, un groupe de travail « Méthodes économiques». Inscrite dans la perspective du développement durable, l'intention initiale est également de pouvoir tirer parti des travaux touchant aux aspects économiques de cette problématique, notamment pour essayer d'en extraire des éclairages susceptibles de mieux guider l'intervention publique ${ }^{28}$. Là aussi, la tendance est à élever la mobilisation de la discipline économique au rang de nécessité, comme va d'ailleurs le faire Christian Brodhag, en tant que Président de la CFDD : «Que ce soit la prise en compte du long terme, et donc le rôle du taux d'actualisation, que l'usage des outils économiques, la science économique est amenée à apporter sa contribution dans la gouvernance.» (CFDD [1997, p 8]).

Les responsables de la D4E vont d'ailleurs marquer rapidement un intérêt dans le maintien de relations relativement informelles avec un ensemble d'économistes qui peut aussi leur servir de vivier. Cette volonté d'entretenir un réseau conduit notamment à un effort d'écriture et de publication, conçu comme un moyen de conserver une capacité de discussion. Les liens ainsi entretenus prennent une importance particulière lorsque se fait sentir le besoin d'un avis scientifique: dans ces situations, ces responsables ont d'abord tendance à aller chercher des spécialistes qu'ils connaissent et dont ils pensent pouvoir mettre à contribution les compétences.

\section{Un soutien institutionnel à la recherche en économie de l'environnement}

Dans tout ce processus d'institutionnalisation, l'intérêt des services de l'État pour l'économie de l'environnement ne se manifeste pas seulement par la mobilisation de travaux déjà réalisés, mais aussi de plus en plus par l'élaboration de commandes utilitaires et par un soutien, notamment financier, à la recherche dans ce domaine. Cet effort institutionnel prend surtout de l'importance à partir du début des années 1990, à un moment où sont plus souvent mises au jour les déficiences du potentiel de recherches économiques sur les thèmes environnementaux. L'insuffisance du développement de l'économie de l'environnement est un constat qui est, par exemple, formulé au colloque Environnementéconomie»

28. Le mandat du groupe est présenté dans : Commission française du développement durable [1997, p. 104]. La liste des participants du groupe figure p. 105-106. Pour la présentation des réflexions, voir Commission française du développement durable/Commissariat général du Plan [1997]. 
tenu les 15 et 16 février 1993 sous l'égide du ministère de l'Environnement, de l'IFEN et de l'INSEE 29 .

Ce champ de recherche va ainsi bénéficier d'une attention nettement plus marquée au ministère de l'Environnement, et il tend même à se voir confier un rôle primordial. Dans la perspective du service de la Recherche et des Affaires économiques, c'est un secteur qui mérite une place particulière, à la mesure de l'appoint qu'il pourrait fournir à la régulation publique : «L'économie constitue une priorité pour le ministère de l'Environnement, avec pour objet d'identifier, de soutenir et de développer les équipes de recherche universitaires capables d'aider à la mise au point d'outils et de méthodes utiles pour la politique de l'environnement aux plans national et local, et au plan international ${ }^{30}$. » La ligne de crédits pour la recherche en économie apparait au ministère de l'Environnement en 1993, et elle va bénéficier d'une augmentation rapide ${ }^{31}$. L'effort de structuration de ce champ de recherche est poursuivi en 1995 avec la création, par ce même ministère, du Programme interinstitutionnel de recherches et d'études en économie de l'environnement (PIREE). Outre les travaux financés, ce programme a donné lieu à des "Journées Économie de l'environnement du PIREE » à Strasbourg les 2 et 3 décembre 1999, et à un colloque sur Les instruments des politiques environnementales à Sophia-Antipolis les 5 et 6 avril 2001. Ces conférences offrent à nouveau des lieux d'échange où des acteurs du monde scientifique et académique peuvent côtoyer des responsables administratifs intéressés par ces approches économiques.

Dans le cadre de ses missions, l'ADEME a aussi entrepris le financement de programmes de recherche touchant à l'économie de l'énergie et de l'environnement. Le type d'objectifs affichés par l'organisme pour ce domaine est similaire, puisqu'il s'agit de «structurer le potentiel de recherche » et de "promouvoir la capacité d'expertise française vis-à-vis des enjeux et débats internationaux ${ }^{32}$.»

La nouvelle D4E du ministère de l'Environnement a continué à encourager le développement des investigations scientifiques dans le domaine économique. Dans le prolongement du PIREE et avec le souci de soutenir des démarches finalisées, un nouveau programme de recherche, baptisé «Sciences économiques et environnement» (S3E), a été lancé au printemps 2002 sous l'égide du ministère, pour que «la recherche en économie de l'environnement puisse éclairer ses travaux d'évaluation économique des politiques environnementales et

29. Jacques Theys [1993, p. 44-45] avance alors trois raisons pour expliquer la «faiblesse chronique des recherches et études économiques sur l'environnement »: " un système public de décision qui, globalement, utilisait peu les études économiques dans le domaine de l'environnement », « la marginalisation de l'économie de l'environnement au sein du système universitaire », et «l'insuffisance des informations de base. »

30. «Les crédits de recherche », ministère de l'Environnement/SRAE [1994, p. 8-9].

31. Elle passe de 0,75 million de francs en 1993 à 4 millions de francs en 1995 (ministère de l'Environnement/SRAE [1995, p. 21]).

32. «Socioéconomie, études, mesures, méthodes », dans ADEME, Programme de recherche et développement 1992-1996, mars 1993, p. 127 
d'intégration de l'environnement dans les politiques sectorielles ${ }^{33}$. » En accordant un soutien contractuel à des chercheurs ou équipes de chercheurs, ces appels d'offre lancés par le ministère de l'Environnement permettent aussi de constituer des réseaux et de structurer une base intellectuelle. Des éléments de ces réseaux peuvent être retrouvés dans les conseils scientifiques qui participent au pilotage et au suivi de ces initiatives de recherche.

S'agissant du soutien à la recherche en économie de l'environnement, il faut aussi signaler le rôle pris par la Commission européenne, notamment avec des lignes de crédits importantes par rapport aux sommes engagées au niveau français. Les programmes ainsi élaborés contribuent, et c'est aussi leur objectif, à renforcer les relations des économistes français intéressés avec leurs homologues des autres États membres.

Au total, il y a non seulement des liens qui se forment entre des communautés travaillant dans des logiques hétérogènes, celles de la recherche économique et celles du travail politico-administratif, mais les relations entre le champ scientifique et le champ bureaucratique tendent aussi à se resserrer. Précisément, les activités que nous venons de décrire permettent l'extension et le renforcement de réseaux en installant de nouvelles connexions. Cette dynamique s'apparente au processus que Michel Callon [1988] et Bruno Latour [1995] désignent par le terme de traduction : celle-ci permet de rapprocher et de coordonner des intérêts auparavant éloignés ou tout au moins hétérogènes, en l'occurrence ceux des producteurs de connaissances économiques et ceux des responsables politiques ou administratifs qui espèrent pouvoir les utiliser.

\section{LES UTILISATIONS INSTITUTIONNELLES DE L'ÉCONOMIE DE L'ENVIRONNEMENT : INTÉRÊTS PRESSENTIS ET BÉNÉFICES ATTENDUS}

Jusqu'à ce point de l'investigation, notre attention était surtout occupée par les processus à travers lesquels des liens se développent entre les détenteurs d'un savoir spécialisé, celui de l'économie de l'environnement, et des acteurs publics de plus en plus intéressés à en faire usage. L'analyse ne pourrait prétendre être complète si nous n'examinions pas sous quelles formes les travaux des économistes sont justement employés.

Des lectures économiques qui peuvent fournir une base argumentaire aux responsables politico-administratifs

Les lectures économiques des responsables publics travaillant sur les questions d'environnement constituent un indicateur utile, non seulement pour

\footnotetext{
33. Direction des Études économiques et de l'évaluation environnementale, Appel à propositions de recherche en économie de l'environnement - Programme S3E (Sciences économiques et environnement), mars 2002.
} 
estimer l'attention que certains travaux ont pu susciter, mais aussi pour saisir l'optique dans laquelle ceux-ci sont abordés ${ }^{34}$. Claude Henry, directeur de recherche au CNRS et professeur à l'École polytechnique (directeur du laboratoire d'économétrie), fait partie de ces économistes dont l'influence laisse des traces dans la littérature politico-administrative : sous la forme de citations, de références en notes de bas de page... Ses travaux ont pu circuler au-delà du milieu des économistes et trouver une audience chez des représentants politiques et administratifs motivés par les préoccupations environnementales. Michel Barnier, alors qu'il est député, y fait par exemple référence dans son rapport parlementaire sur la politique de l'environnement, pour les aspects touchant aux prix des biens d'environnement ${ }^{35}$. Cédric Philibert, ancien chargé de mission au cabinet de Brice Lalonde, s'y réfère également pour rapprocher considérations économiques et éthiques (Philibert [1991, p. 56] ${ }^{36}$.

Pour pousser leur vision de la réalité, les acteurs politiques et administratifs peuvent en fait trouver dans les travaux des économistes sur les questions environnementales un ensemble d'énoncés permettant d'étayer leur argumenta$\operatorname{tion}^{37}$. La démarche s'apparente à une recherche d'alliés, dont la crédibilité peut être mise en avant grâce à l'inscription de leur discours dans le registre scientifi$q^{2} e^{38}$. L'utilisation du répertoire économique peut aussi donner une image de sérieux face à des acteurs restant sceptiques devant les arguments environnementaux.

La nouvelle direction des Études économiques et de l'Évaluation environnementale du ministère de l'Environnement s'efforce d'ailleurs aussi de mettre en visibilité son activité intellectuelle sur les aspects économiques, et elle s'est engagée pour cela dans la publication de «documents de travail», en l'occurrence sous forme de cahiers répartis en trois "séries» («Méthodes», «Études », «Synthèse »). Ces intermédiaires, pensés effectivement comme des dispositifs d'intéressement, matérialisent dans une certaine mesure une volonté de faire circuler des idées et des conceptions, et ce dans un espace qui ne soit pas limité à la seule administration de l'environnement.

34. Nous faisons ici une incursion rapide dans le domaine de la scientométrie et de la bibliométrie (Callon, Courtial, Penan [1993]).

35. Cf. "Pour une réforme des méthodes d'évaluation des grands travaux », dans Rapport d'information $n^{\circ} 1227$, déposé en application de l'article 145 du règlement par M. Michel Barnier, au nom de la commission des Finances, de l'Économie générale et du Plan, sur la politique de l'environnement (11 avril 1990), p. 51.

36. Il cite un article de Claude Henry (École polytechnique, laboratoire d'économétrie, novembre 1989). Ce texte est repris dans Henry [1990].

37. S'agissant des textes placés dans l'espace de discussion politico-administratif, la logique s'avère en fait ici similaire à celle qui imprègne la rédaction des textes scientifiques (Latour [1995], notamment « Faire appel aux amis », p. 82-87, et « Se référer aux textes qui précèdent », p. 87-98).

38. C'est en effet souvent sous la forme d'un «argument d'autorité » que les réflexions d'économistes tendent à apparaitre dans les raisonnements élaborés par les acteurs politiques ou administratifs. Sur cet « appel à des alliés de plus en plus haut placés», cf. Latour [1995, p. 84]. 
Donner un prix à l'environnement : l'insertion de la régulation publique dans une logique de monétarisation

Un des chantiers de l'analyse économique a consisté à développer des outils pour évaluer les atteintes à l'environnement ou les avantages provenant de sa préservation. Les démarches en ce sens, dans la mesure où elles permettent de mettre en visibilité des considérations environnementales, ont pu rencontrer l'intérêt de responsables soucieux de la défense de ce type de considérations. Cette perspective qui gagne en influence tend à faire entrer les problématiques relatives à la qualité de l'environnement ou à sa dégradation dans une logique de mesure basée sur des paramètres économiques.

De fait, dans l'optique dominante chez les économistes, la principale explication de la dégradation de l'environnement et du gaspillage des ressources naturelles est à rechercher dans la "gratuité » de l'utilisation de ce type particulier de biens. Le fait qu'aucun prix ne leur soit donné, qu'ils ne soient pas associés à une valeur économique clairement formulée, éloignerait la collectivité des possibilités d'en faire un usage rationnel. Pour corriger cette situation, des outils et des méthodes pratiques ont donc été élaborés et ont essayé de réintégrer ces biens environnementaux dans un cadre monétaire.

Cette optique est présente assez tôt dans les milieux publics censés assurer la prise en charge institutionnelle des questions d'environnement. Cette absence de prix des biens environnementaux est un problème qui est déjà mis en avant dans les premières années du ministère de l'Environnement par Robert Poujade, le premier titulaire du portefeuille. Ce problème gagne à nouveau une visibilité au tournant des années 1980 et 1990, au moment où les problématiques environnementales reviennent sur le devant de la scène ${ }^{39}$. Michel Barnier, futur ministre de l'Environnement, s'y intéresse à la fin des années 1980. Ses réflexions portent alors sur des sujets comme le "prix du paysage», et elles s'insèrent dans les propositions envisagées pour son rapport parlementaire en préparation. L'enjeu lui apparait en tout cas prioritaire : «L'essentiel, en tout état de cause, est de sortir d'une logique dans laquelle l'environnement est tenu pour gratuit et donc livré à toutes les dilapidations et tous les gaspillages ${ }^{40}$. »

39. Ce type de préoccupation est par exemple exprimé au colloque Écologie et pouvoir de décembre 1989 (Secrétariat d'État auprès du Premier ministre chargé de l'environnement [1990]).

40. "Pour une réforme des méthodes d'évaluation des grands travaux", dans Rapport d'information $\mathrm{n}^{\circ} 1227$, déposé en application de l'article 145 du règlement par M. Michel Barnier, au nom de la commission des Finances, de l'Économie générale et du Plan, sur la politique de l'environnement (11 avril 1990), p. 51. 
Cette optique tend d'ailleurs à devenir d'autant plus prégnante qu'elle bénéficie aussi de l'appui de pôles d'expertise influents comme l'OCDE ${ }^{41}$.

Dans cette approche, l'ensemble des conséquences économiques résultant de l'utilisation de l'environnement doit donc pouvoir être mis en visibilité par le développement d'un travail de monétarisation. C'est cette perspective qui peut être retrouvée dans les ouvrages édités annuellement par le ministère de l'Environnement sous le titre Données économiques de l'environnement, et c'est pour une large part dans ce registre monétaire de mise en visibilité que tendent à être positionnées les parties intitulées "Évaluation des dommages », puis «L'économie des pressions ».

Au total, le développement des travaux sur le recours aux systèmes de prix a pu permettre de formuler des argumentaires justificatifs, propres à intéresser des responsables publics à l'exploration de ce nouveau registre d'intervention. Malgré des méthodes d'évaluation qui ne sont pas encore stabilisées, et qui restent même parfois fortement controversées, c'est une voie vue sous un jour prometteur, notamment pour corriger les usages les plus insouciants des ressources naturelles. La quantification monétaire tend alors à faire figure de cadre universellement valable pour saisir les dysfonctionnements dans l'utilisation collective des biens environnementaux et, de manière plus pratique, pour les réduire en les réarticulant à l'ordre économique. Surtout, dans ce mouvement, la monétarisation ne joue pas simplement comme méthode d'évaluation, mais aussi de plus en plus comme répertoire de principes directifs pour la prise de décision.

\section{Attraits du calcul économique et poids des analyses coûts-avantages}

Le calcul économique et l'analyse coûts-avantages figurent couramment en bonne place dans le répertoire des outils d'aide à la décision. D'ailleurs, les développements que ces méthodes économiques ont connus, notamment sous l'impulsion d'une lignée d'ingénieurs-économistes, ne sont pas spécialement liés aux questions d'environnement ${ }^{42}$. Celles-ci constituent en fait un des domaines

41. La position de l'OCDE insiste en fait sur la nécessité de déterminer non seulement un prix pour les biens environnementaux, mais aussi de fixer ce prix à un niveau correct : «L'établissement du juste prix est probablement le plus important facteur dans la mise en œuvre effective d'un développement durable. Tant que le prix des matières premières et des produits ne reflétera pas de façon satisfaisante les coûts sociaux et qu'un prix ne pourra être affecté à l'eau, à l'air et aux ressources en sols, actuellement utilisés à titre gratuit en tant qu'intrants ou en guise de réceptacles des déchets produits par la société, l'allocation des ressources sera inefficace et la pollution de l'environnement aura toutes les chances de s'aggraver. Malheureusement, par le passé, le prix de nombreuses ressources naturelles est resté fixé à un niveau trop bas pour refléter la totalité de leur coût. Qui plus est, la tarification des ressources ne tient généralement pas compte des coûts associés à leur consommation et à leur éventuel épuisement. » (OCDE [1992, p. 16].)

42. S'agissant du calcul économique, son histoire remonte bien plus loin que les décennies où ont émergé les préoccupations environnementales (Etner [1987].) 
d'application possibles ${ }^{43}$, en l'occurrence un domaine qui a pu prendre de l'extension grâce à la plus grande visibilité des préoccupations écologiques. Des ajustements ont ainsi été concédés dans les pratiques sur lesquelles se fondaient le calcul économique et l'analyse coûts-avantages : il s'agissait de pouvoir mieux prendre en compte des conséquences se situant au-delà du court terme, d'essayer d'intégrer les effets externes environnementaux, de faire apparaître le poids de l'incertitude dans certains cas.

Les tentatives dans ces différentes directions s'apparentent davantage à un prolongement qu'à un déplacement des orientations préexistantes dans ces méthodes. Le calcul économique est utilisé pour évaluer des projets et sélectionner ceux qui pourront être les plus profitables à la collectivité. Il reste axé sur la mesure de la rentabilité économique et de l'utilité sociale des projets présentés. De même, c'est la recherche de l'efficience qui sous-tend l'analyse coûtsavantages et qui doit permettre de déterminer l'allocation de ressources censée maximiser le bien-être collectif ${ }^{44}$. Ces méthodes économiques agrègent ainsi un large ensemble d'intérêts parce qu'elles laissent en somme espérer la révélation d'une situation d'optimum.

L'essentiel des attentes dans les milieux publics intéressés continue à se porter sur la possibilité d'éclairer les choix publics en recourant à ces évaluations économiques des coûts et bénéfices sociaux, au besoin aménagées pour tenir compte des contraintes environnementales. La comparaison de ces éléments sous une forme monétaire offre une voie permettant de produire des critères de choix, de surcroît dans un cadre en apparence simplifié. À partir de la quantification et de la possible mise en balance des projets avec leurs effets environnementaux se développent ainsi des arguments donnant à voir ces méthodes, audelà de leurs différences d'approches, comme le moyen le plus approprié d'approcher la réalité. L'analyse coûts-avantages trouve d'ailleurs des partisans dans les défenseurs de la cause environnementale, qui pensent grâce à cela pouvoir convaincre les sceptiques, notamment en montrant que leur cause peut aussi entrer dans le registre du calculable, avoir une expression monétaire, et donc trouver une légitimité parmi des logiques d'action déjà installées.

Les recommandations dans cette direction n'excluent pas pour autant les critiques. Dominique Dron et Michel Cohen de Lara, dans le cadre de leurs activités à la cellule " Prospective et stratégie » du ministère de l'Environnement, en ont soulevé un certain nombre dans leur rapport intitulé Évaluation économique et environnement dans les décisions publiques ${ }^{45}$. Le rapport signale des usages contestables du calcul économique et essaye de réduire certaines prétentions expansives

43. Pour une présentation (méthodes, limites...), voir Faucheux, Noël [1995, p. 219 et sq.].

44. Ceci peut aboutir à des raisonnements aussi surprenants que réducteurs, comme celui de Georges Honoré à propos de l'effet de serre : «L'impact économique d'un changement climatique, faible dans les zones équatoriales saturées de vapeur d'eau, pourrait être positif dans les zones tempérées, les surcoûts agricoles (irrigation...) étant probablement compensés par des gains touristiques. » («Comptes des transports : 25 milliards de francs de surcoût des externalités de la route », Notes de synthèse OEST, octobre 1993.)

45. Rapport au ministre de l'Environnement, Paris, La Documentation française [1998]. 
présentes dans le recours à ce type de méthode. Le document souligne en outre le décalage entre la forte présence du calcul économique dans les argumentaires institutionnels et une rigueur dont la solidité est plus difficile à constater dans le déroulement des processus décisionnels. C'est une situation que le ministère de l'Environnement avait plutôt tendance à subir, parce que, dans les jeux administratifs où ces méthodes économiques sont engagées, il ne disposait pas des ressources, notamment en termes de compétences, lui permettant d'examiner et de mettre sérieusement en doute les résultats produits. La nouvelle D4E a été pour partie positionnée pour corriger cette faiblesse.

Des efforts d'intégration des facteurs environnementaux dans les modélisations économiques

Les travaux dans le domaine de la modélisation macroéconomique ont aussi bénéficié d'un soutien plus marqué pour pouvoir mieux prendre en compte les relations entre dimensions économiques et environnementales à un niveau global. Dans ce domaine, un courant d'attention peut ainsi être repéré au ministère de l'Environnement. Au début des années 1980, un modèle dénommé POLLEN a été développé au sein du CEPREMAP par Michel Manuel pour le groupe de prospective du ministère. Des travaux ont également été engagés plus tard pour le ministère par l'équipe "DIVA» de la société de conseil BIPE. C'est avec ce modèle DIVA - Dynamique Intersectoriel à Vingt Ans - que cette société a réalisé une évaluation de l'impact économique du Plan national pour l'environnement lancé sous l'égide de Brice Lalonde.

En France, les travaux effectués par l'Équipe de recherche analyse des systèmes et modélisation économique (ERASME, École centrale et Université Paris I) du professeur Paul Zagamé ont pris une place importante dans le domaine de la modélisation. C'est d'ailleurs lui et Olivier Beaumais, chercheur au sein de cette équipe, qui font le bilan de ce champ d'étude lors du colloque Environnement économie de février 1993 (Beaumais, Zagamé [1993].)

Des modèles baptisés MIDAS et HERMES ont par exemple été combinés par l'équipe ERASME pour évaluer les effets macroéconomiques du projet de taxe $\mathrm{CO}_{2}$ /énergie avancé en 1991 par la Communauté européenne. À partir de ce système de modèles économétriques de grande taille, composant une description macrosectorielle de l'économie et du système énergétique, ont pu être produits des résultats qui ont nourri les travaux engagés dans le cadre de la commission «Environnement, qualité de vie, croissance » du XIe Plan. Ce projet de taxation était en effet discuté pour ses effets possibles sur la compétitivité. La réalisation d'une telle évaluation rejoignait en particulier a priori les intérêts des industriels présents dans cette commission, et notamment de son président Bertrand Collomb, PDG du groupe cimentier Lafarge Coppée ${ }^{46}$.

46. A priori, car les résultats de ces travaux se sont avérés éloignés de ceux espérés par les représentants des milieux industriels et ont donc été critiqués et rejetés au profit d'autres travaux, apparemment plus conformes à leur vision de la situation. 
Ces travaux de modélisation tentant d'intégrer les aspects environnementaux ont pu se développer grâce à un renforcement de l'appui des institutions nationales et européennes. Du côté français, le ministère de l'Environnement, l'ADEME, le Commissariat général du Plan, et la chambre de commerce et d'industrie de Paris y ont pris part de manière active. Au niveau communautaire, des réseaux de recherche ont pu se structurer grâce au soutien de la commission, qui a ainsi pu trouver là des capacités d'expertise plus solides, fort utiles notamment pour certaines négociations internationales.

Dans le dossier de l'effet de serre, des modèles macroéconomiques appliqués ont par exemple été mis à contribution pour fournir des évaluations sur les effets des mesures correctives envisagées ${ }^{47}$. En France, des démarches ont ainsi été engagées pour apprécier les répercussions économiques potentielles des engagements de réduction des émissions de gaz à effet de serre pris lors du protocole de Kyoto de décembre 1997. Les évaluations ont été en l'occurrence effectuées à l'aide de GEMINI-E3, modèle d'équilibre général développé par le ministère de l'Équipement et le Commissariat à l'énergie atomique, et de POLES, modèle d'équilibre partiel du secteur énergétique mondial, développé par l'Institut d'économie et de politique de l'énergie - Unité mixte de recherche du CNRS et de l'Université Pierre Mendès-France de Grenoble.

\section{Développement et affinement d'un corps d'instruments économiques dans une optique de régulation environnementale}

Les arguments mis en forme dans le langage de la science économique ont aussi pris une place croissante dans les débats politico-administratifs concernant la conception et l'utilisation d'instruments d'action publique applicables aux enjeux environnementaux. Ces débats ont traduit des souhaits de plus en plus répandus d'élargir le répertoire des instruments utilisés, notamment en explorant des solutions sortant de l'outillage réglementaire et administratif qui a longtemps structuré l'intervention étatique en matière d'environnement.

De manière symptomatique, l'argument d'efficacité, qualité donnée le plus souvent a priori, tient une place importante dans l'espace de discussion ainsi formé autour des instruments dits économiques, et en particulier des taxes et des permis d'émission négociables. À ces instruments économiques, incitatifs dans le sens où ils font jouer des mécanismes de prix, tendent en effet à être

\footnotetext{
47. Sur les logiques et controverses présentes dans les lieux d'expertise à propos de l'effet de serre, voir le point de vue interne d'Olivier Godard, «L'expertise économique du changement climatique planétaire - 1. Modèles d'organisation de l'expertise (p. 23-28). 2. Sur la scène internationale, le GIEC (p. 29-50). 3. Sur la scène française, autour du Commissariat général du Plan (p. 51-65) ", dans Annales des Mines - Série Responsabilité et environnement, n² 21, janvier [2001].
} 
couramment associées des propriétés d'efficacité économique ${ }^{48}$. La logique à l'œuvre dans ce schéma d'appréhension se prolonge souvent par une remise en cause des solutions en vigueur : privilégiant ordinairement les voies réglementaires et administratives, elles tendent à être critiquées pour leur insuffisance sur le plan incitatif, et même soupçonnées d'être une source de dysfonctionnements et d'inefficacités. La solution des instruments économiques tend, en revanche, à être présentée comme un moyen de remédier aux inconvénients des approches plus traditionnelles: par le jeu des dynamiques incitatives, elle doit permettre de réaliser les objectifs environnementaux en minimisant, par la même occasion, les coûts supportés par la collectivité.

Dans ce registre, les travaux engagés sous l'égide de l'OCDE sur les instruments économiques ont pu fournir tout un répertoire de justifications. Le recours à ce type d'instrumentation est en effet une approche pour laquelle cette organisation internationale se distingue en ayant consenti un effort important et constant, à la fois sur le plan de la recherche et des recommandations. Sur cet aspect également, ouvrages et guides pratiques sont ainsi venus régulièrement compléter le catalogue de références éditées par l'OCDE (OCDE [1991] ; [1994]), incluant au fur et à mesure les avancées que les travaux d'économistes semblaient produire. Le même corps central d'arguments et de recommandations y est généralement mis en avant pour promouvoir une utilisation plus large de ces instruments économiques, ces derniers étant en l'occurrence censés offrir plus de souplesse, favoriser une incitation permanente et réduire les coûts de manière significative. Dans de nombreux discours politiques et administratifs sur la question, c'est aussi couramment la description de ces avantages qui est reprise et qui vient fonder les principaux espoirs affichés.

En France, et plus fréquemment depuis le début des années 1990, les propositions d'instruments économiques avancées par les autorités publiques ont aussi entraîné dans leur sillage des séries de travaux, destinés pour une large part à vérifier la pertinence et les effets des solutions préconisées. Ce fut le cas avec la taxe mixte carbone-énergie proposée par la Commission européenne en septembre 1991 afin de limiter les émissions de $\mathrm{CO}_{2}$. Les argumentations et contre-argumentations déployées ont pu recourir aux projections effectuées sur les conséquences de ce type de mesure au plan microéconomique comme au plan macroéconomique. En liaison pour partie avec ce contexte, la composante fiscale est aussi présente dans les réflexions engagées dans le cadre du XIe Plan.

Les années qui suivent vont voir les débats se développer autour du renforcement d'une «fiscalité écologique ». Différents rapports et études y participent,

48. De manière symptomatique également, c'est un registre de justification particulièrement présent dans le compte rendu des travaux de l'atelier "Environnement, économie, croissance », majoritairement composé d'économistes, de la Commission «Environnement, qualité de vie, croissance » du XIe Plan : « Néanmoins, en l'état actuel des instruments utilisés, il parait souhaitable d'accroître le recours aux instruments économiques ; en particulier le recours aux redevances, péages et taxes à finalité incitative devrait être plus fréquemment envisagé, tant en raison de leurs propriétés d'efficacité économique que de leur capacité à constituer un contexte plus favorable à l'action des autres types d'instruments » (Commissariat général du Plan [1993b, p. 105].) 
avec plus ou moins d'influence, en complétant le répertoire des arguments disponibles, notamment quant aux carences à combler ou aux ajustements à effectuer dans les dispositifs fiscaux. Les travaux et débats organisés en 1998 dans le cadre du Conseil d'analyse économique sur la «réforme de la fiscalité de l'environnement» ont ainsi constitué un moment particulièrement visible, en faisant en l'occurrence à nouveau intervenir un réseau de personnalités déjà solidement positionnées dans le champ disciplinaire de l'économie de l'environnement (Conseil d'analyse économique [1998]). Logiquement, la circulation des références économiques a trouvé un écho dans les discours qui ont participé à la promotion de la taxe générale sur les activités polluantes (TGAP), celle-ci étant par exemple censée jouer un rôle incitatif grâce à l'émission d'un « signal-prix ». Les projets de renforcement du pan environnemental de la fiscalité ont aussi fourni l'occasion de poursuivre les investigations autour de l'argument du «double dividende», autrement dit l'argument d'une taxe pouvant procurer conjointement des avantages environnementaux et des retombées positives en matière d'emploi.

Un autre champ de réflexions et d'études a été ouvert par la proposition d'un système international de permis d'émission négociables pour la gestion préventive du réchauffement climatique. La mise en place d'un tel système, dans le sillage du protocole de Kyoto, suppose que soit défini et accepté un ensemble de règles communes. D'où l'émergence, à la fois dans le champ académique et dans le champ politico-administratif, de toute une gamme d'interrogations plus ou moins précises sur les modalités de conception et de fonctionnement de telles règles. L'aide des économistes a là aussi été recherchée pour combler ce besoin de connaissances, non seulement pour évaluer les expériences existantes sur ce type d'instrument, mais aussi et surtout pour envisager de manière prospective et chiffrée le jeu des répercussions potentielles, notamment en fonction des hypothèses et objectifs mis en débat ${ }^{49}$. Les résultats d'études trouvent ainsi particulièrement leur utilité dans le cadre de négociations internationales qui peuvent déboucher sur des engagements contraignants, l'appréciation des coûts induits apparaissant alors effectivement comme un enjeu important. Tout en prolongeant les travaux dans le domaine des émissions atmosphériques, la D4E du ministère de l'Écologie et du Développement durable a d'ailleurs aussi continué à explorer les solutions d'internalisation recourant aux marchés de permis, en essayant d'étudier l'extension de cette option à d'autres domaines, comme la gestion des excédents de nitrates en Bretagne et celle du bruit autour des aéroports.

Au vu de ces courants de démarches plus ou moins liés entre eux, le travail institutionnel dans le domaine de l'environnement montre donc le développement d'un ensemble de technologies intellectuelles assises sur les avancées de la science économique. Les responsables publics, qui ont des décisions à prendre

49. Le rapport remis par Roger Guesnerie dans le cadre du Conseil d'analyse économique est caractéristique de ce type de démarche, utilisant de surcroît en l'occurrence une volonté de synthèse pour ouvrir vers un cadre de propositions (Conseil d'analyse économique [2003]). 
ou des options à justifier, cherchent plus fréquemment un soutien du côté des outils économiques disponibles et tendent même au besoin à en faciliter le perfectionnement. Cela vaut, comme on vient de le voir, pour les évaluations monétaires, les calculs de type coûts-avantages, ou les modèles économiques plus ou moins raffinés. Conjointement, l'appareillage technique offert par la science économique tend à être présenté comme le support d'un mouvement devant permettre de rationaliser la prise en charge collective des problèmes.

\section{CONCLUSION}

Les argumentations institutionnelles développées autour des problématiques environnementales laissent transparaître une influence de plus en plus sensible de considérations, de références, qui peuvent être plus ou moins directement rattachées au champ de la science économique et à ses prolongements. Certes, il ne s'agit pas d'un envahissement massif. Toutefois, dans les milieux publics concernés, ces problématiques environnementales apparaissent de plus en plus souvent mises en forme en des termes empruntant une part notable de leur substance au langage économique.

Précisément, le développement de l'intervention étatique dans le domaine environnemental a amené les responsables intéressés à rechercher des appareillages intellectuels appropriés, capables d'asseoir et de donner à voir une maitrise à terme des problèmes soulevés. Les développements conceptuels réalisés dans le champ de la science économique s'offrent comme des instruments intellectuels devant permettre de mieux prendre en compte les aspects de la nature, et ce en guidant convenablement le comportement des hommes. Dans ce schéma, la prise en charge institutionnelle de l'environnement tend à se trouver construite comme un problème technique pour lequel la participation des professionnels de l'économie devient de plus en plus essentielle. De ce point de vue, les outils de l'analyse économique constituent des pièces participant à un processus de rationalisation, lui-même en congruence idéologique avec une montée des enjeux d'efficacité économique. Face aux problèmes qu'ils ont à traiter, les responsables publics font plus largement appel au savoir spécialisé des économistes pour trouver des propositions transposables. Si les professionnels de l'économie de l'environnement voient leurs compétences reconnues, il faut toutefois préciser que leurs observations et réflexions bénéficient surtout d'une attention pour des questions qui sont déjà au centre de l'espace de discussion politico-administratif.

De fait, il y a un penchant instrumentaliste dans l'utilisation que les responsables publics intéressés peuvent faire de l'économie de l'environnement. Comme l'ont souligné les analyses de Giandomenico Majone [1989], les productions relevant de la science économique sont suffisamment diverses pour fournir aux responsables politico-administratifs un répertoire d'arguments dans lequel puiser selon leurs besoins. C'est ce qui peut expliquer que les critiques et 
regrets des économistes sont fréquents lorsqu'ils peuvent juger de l'importation et de la retranscription de leurs conceptions et argumentations dans l'espace de discussion politico-administratif : ceux qui approchent cette sphère ont souvent le sentiment que leurs recommandations sont négligées ou déformées par les acteurs en position décisionnelle. Les analyses produites dans le registre scientifique tendent par ailleurs à entrer dans un jeu dans lequel elles deviennent un moyen de légitimer telle ou telle proposition politique, ou de disqualifier telle autre. Plutôt que d'éclairer les décideurs, les analyses économiques peuvent alors servir à produire des justifications a posteriori pour des choix déjà effectués.

Par conséquent, il faut faire sérieusement attention à la logique sociale dans laquelle s'insère le recours à l'économie de l'environnement. En étant intégrées dans les répertoires argumentaires institutionnels, les analyses de cette branche disciplinaire peuvent, comme celles d'autres disciplines d'ailleurs, introduire des effets de cadrage dans les processus décisionnels. Les schémas d'analyse utilisés peuvent en effet orienter profondément les propositions venant dans l'espace de discussion. Sous couleur d'aider à appréhender les problèmes et à les prendre en charge, le recours à une optique particulière comme celle de l'économie peut conduire implicitement à un tri dans la gamme des possibilités envisagées.

De ce point de vue, la question des soubassements normatifs de ces perspectives économiques est donc loin d'être négligeable. Précisément, les cadres théoriques et analytiques mis à contribution ne sont pas sans arrière-plan social : ils contiennent de manière plus ou moins visible certaines conceptions de la société, des acteurs qui la composent, de son rapport à la nature. La plupart du temps, les propositions tirées du savoir économique s'avèrent ainsi soustendues par des hypothèses dont l'explicitation n'est guère assurée ou dont le caractère discutable et controversé est mis en retrait. S'agissant des efforts de gestion de l'environnement, c'est typiquement la situation vers laquelle penchent les démarches visant à comparer des coûts et des bénéfices : celles-ci comportent des choix intellectuels pas toujours clairement formulés, mais qui ont leur importance pour les résultats produits.

Au surplus, l'interprétation dans le registre monétaire tend à modifier les modalités d'appréhension des questions d'environnement: la logique de l'internalisation des coûts externes fait de ces questions une affaire de mécanique économique, ce qui peut conduire à l'effacement de certains facteurs relevant de l'ordre politique et justiciables d'une discussion collective. Dans cette logique d'internalisation, c'est en effet aussi le jeu des responsabilités qui tend à être redistribué : à suivre cette orientation, les comportements dommageables ne s'expliqueraient que par le fait que les agents économiques ne reçoivent pas les bons signaux en termes de prix. Pour corriger ces comportements, le registre de l'injonction plus ou moins morale, éventuellement prolongée par la sanction législative, devrait alors céder la place à celui de l'incitation économique. En définitive, c'est le positionnement de la régulation publique qui peut ainsi se trouver déplacé par l'adaptation institutionnelle de la pensée économique aux enjeux environnementaux. 


\section{RÉFÉRENCES BIBLIOGRAPHIQUES}

ACTES DE LA RECHERCHE EN SCIENCES SOCIALES (1997), «Économie et économistes », $\mathrm{n}^{\circ} 119$, septembre.

Akrich Madeleine, CALlon Michel, Latour Bruno (1988), « À quoi tient le succès des innovations. Premier épisode : L'art de l'intéressement», Gérer et comprendre, n 11, juin.

AYRES Robert, KNEESE Allan (1969), "Production, consumption and externalities », American Economic Review, 59, juin.

BEAUMAIS Olivier, SCHUBERT Katheline (1996), "Modèles d'équilibre général appliqués à l'environnement: développements récents ", Revue d'économie politique, 106 (3), mai-juin.

Beaumais Olivier et Zagamé Paul (1993), «Bilan de l'apport des modèles à l'évaluation économique des politiques d'environnement », dans INSEE/IFEN/Ministère de l'Environnement, Environnement économie, Actes du colloque, Paris, 15 et 16 février 1993, INSEE Méthodes n 39-40, Paris, INSEE.

BOULDING Kenneth (1966), "The economics of the coming spaceship earth ", dans Henry JARRETT (ed.), Environmental Quality in a Growing Economy, Baltimore, John Hopkins University Press.

Bureau Dominique, Godard Olivier, Hourcade Jean-Charles, Henry Claude, LIPIETZ Alain (1998), Fiscalité de l'environnement, Rapports du Conseil d'analyse économique, Commentaires Jean-Philippe CoTis, Michel Mougeot, Paris, La Documentation française.

CALLON Michel (dir.) (1988), La science et ses réseaux. Genèse et circulation des faits scientifiques. Paris : La Découverte/Conseil de l'Europe/Unesco.

CALlON Michel (ed.) (1998), The Laws of the Markets, Oxford, Blackwell Publishers.

Callon Michel, Courtial Jean-Pierre, Penan Hervé (1993), La scientométrie, Paris, PUF, coll. «Que sais-je ?» n² 2727.

Callon Michel, Latour Bruno (1997), "'Tu ne calculeras pas!' ou comment symétriser le don et le capital », Revue du MAUSS, n 9, premier semestre.

COMMISSARIAT GÉNÉRAL DU PlAN (1993a), Environnement, qualité de vie, croissance. Rapport des ateliers, octobre.

Commissariat GÉnÉral du Plan (1993b), L'économie face à l'écologie, Paris, La Découverte/La Documentation française.

COMMISSION FRANÇAISE DU DÉVELOPPEMENT DURABLE (1997), De la prise de conscience vers la mobilisation, Rapport 1997.

COMMISSION FRANÇAISE DU DÉVELOPPEMENT GURABLE/COMMISSARIAT GÉNÉRAL DU PLAN (1997), Approches économiques du développement durable, Rapport du groupe de travail de la commission (Synthèse du rapport intérimaire), Les cabiers $d u$ développement durable, $\mathrm{n}^{\circ} 5$, décembre.

CONSEIL D'AnAlyse ÉCONOMiQue (2003), Kyoto et l'économie de l'effet de serre, Paris, La Documentation française.

DRON Dominique, COHEN DE LARA Michel (cellule « Prospective et stratégie ») (1995), Évaluation économique et environnement dans les décisions publiques. Rapport au ministre de l'environnement, Paris, La Documentation française.

ETNER François (1987), Histoire du calcul économique en France, Paris, Economica.

FAUCHEUX Sylvie, NOËL Jean-François (1995), Économie des ressources naturelles et de l'environnement, Paris, Armand Colin. 
Gastaldo Sylviane, Mazodier Pascal, Prevot Henri (1998), «Le développement de l'approche économique au ministère de l'Aménagement du territoire et de l'Environnement : une nécessité fructueuse », Rapport de la mission sur "Le renforcement des capacités d'expertise économique du ministère de l'Aménagement du territoire et de l'Environnement pour l'évaluation des politiques publiques gouvernementales et l'aide à la décision », 15 octobre.

GODARD Olivier (1998), «Les sciences économiques et les recherches sur l'environnement », dans La question de l'environnement dans les sciences sociales. Éléments pour un bilan, Programme Environnement, Vie et Société du CNRS, Lettre $\mathrm{n}^{\circ} 17$, février, numéro spécial.

GODARD Olivier (2001), "L'expertise économique du changement climatique planétaire », Annales des Mines - Série Responsabilité et environnement, $\mathrm{n}^{\circ} 21$, janvier.

GROUPE INTERMINISTÉRIEL D'ÉVALUATION DE L'ENVIRONNEMENT (1975), Méthodologie et théorie économique de l'environnement, Paris, La Documentation française.

GuERrien Bernard (1996), "Néo-classique (théorie)», dans Dictionnaire d'analyse économique, Paris, La Découverte, p. 352-355.

HAAS Peter M. (1992), "Introduction : epistemic communities and international policy coordination", International Organization, 46 (1), hiver.

HABERMAS Jürgen (1973a), La technique et la science comme «idéologie », Paris, Gallimard.

HABERMAS Jürgen (1973b), «Scientifisation de la politique et opinion publique », dans La technique et la science comme «idéologie», Paris, Gallimard.

HENRY Claude (1990), «Efficacité économique et impératifs éthiques: l'environnement en copropriété », Revue économique, 41 (2), mars.

INSEE/IFEN/Ministère de l'Environnement (1993), Environnement économie, Actes du colloque, Paris, 15 et 16 février, INSEE Méthodes, n 39-40, Paris, INSEE.

JACOBS Michael (1994), "The limits to neoclassicism. Towards an institutional environmental economics", dans REDCLIFT Michael and BENTON Ted (eds), Social Theory and the Global Environment, Londres, Routledge.

JOBERT Bruno (1993), "Ambiguité, bricolages et modélisation. La construction intellectuelle des politiques publiques ", dans CRESAL, Les raisons de l'action publique (Entre expertise et débat), Paris, L'Harmattan.

JOBERT Bruno (dir.) (1994), Le tournant néo-libéral en Europe, Paris, L'Harmattan. Seuil.

JulliarD Jacques et WINOCK Michel (1996), Dictionnaire des intellectuels français, Paris,

KRUTILla John (1967), «Conservation reconsidered », American Economic Review, 57 (4), septembre.

LARRUE Corinne (1995), "The Political (Un)feasibility of Environmental Economic Instruments ", dans DENTE Bruno (ed.), Environmental Policy in Search of New Instruments, Dordrecht, Kluwer Academic Publishers.

LATOUR Bruno (1995), La science en action, Paris, Gallimard, coll. Folio/Essais.

LEPAGE Corinne (2000), « Le ministère de l'Environnement est-il en passe de devenir l'annexe du ministère des Finances ? ", Valeurs vertes, janvier-février.

LIPIETZ Alain (1992), «Ecologie : une logique économique post-socialiste », La Nouvelle Revue socialiste $», \mathrm{n}^{\circ} 17$, juillet.

LORDON Frédéric (1997), "Le désir de 'faire science'», Actes de la recherche en sciences sociales, $\mathrm{n}^{\circ} 119$, septembre.

Majone Giandomenico (1989), Evidence, Argument and Persuasion in the Policy Process, New Haven CT, Yale University Press.

MALINVAUD Edmond (1997), La fonction statistique et études économiques dans les services de l'État, Rapport au Premier ministre, janvier. 
Miller Peter, Rose Nikolas (1990), « Governing economic life », Economy and Society, 19 (1), février.

Ministère De L’ÉCONOMIE ET Des FinanCes (1992), Les Notes Bleues nº63, «Croissance et environnement », 27 juillet-2 août.

Ministère DE L'EnVIRONNEMENT/SRAE (1994), BRISE (Bulletin de Recherche et d'Information scientifique sur l'environnement), $\mathrm{n}^{\circ} 0$, décembre.

Ministère DE L'EnVIRONNEMENT/SRAE (1995), BRISE (Bulletin de Recherche et d'Information scientifique sur l'environnement), $\mathrm{n}^{\circ} 1$.

OCDE (1992), Environnement et économie : Les travaux de l'OCDE, Paris, OCDE.

OCDE (1994), Gérer l'environnement : le rôle des instruments économiques, Paris, OCDE.

OCDE (1995), Évaluation économique des politiques et projets environnementaux. Un guide pratique, Paris, OCDE.

OCDE (1997), Examen des performances environnementales. France, Paris, OCDE.

PASSET René (1979), L'Économique et le Vivant (1 ${ }^{\text {re }}$ édition ; réédition, Paris, Economica, 1996).

PHILIBERT Cédric (1991), «Et l'écologie, par-dessus le marché ? », Esprit, n 174, septembre.

RUMPALA Yannick (2003), Régulation publique et environnement. Questions écologiques, réponses économiques, Paris, L'Harmattan.

SECRÉtARIAT D'ÉtAT AUPRÈs DU PREMIER Ministre CHARgÉ DE L'ENVIRONNEMENT (1990), Écologie et pouvoir, Colloque 13, 14, 15 décembre 1989, Paris, La Documentation française.

SPASH Clive L. (1999), "The Development of Environmental Thinking in Economics », Environmental Values, 8 (4).

THEYS Jacques (1993), «Environnement et économie : quels axes pour la recherche dans les années à venir ", dans INSEE/IFEN/ministère de l'Environnement, Environnement économie, Actes du colloque, Paris, 15 et 16 février 1993, INSEE Méthodes nº 39-40, Paris, INSEE.

VIVIEN Franck-Dominique (1994), Économie et écologie, coll. "Repères», Paris, La Découverte.

VIVIEN Franck-Dominique (1997), «L'économie et l'écologie entre science et idéologie », Natures Sciences Sociétés, 5 (4), octobre-décembre.

WISMAN Jon D. (1990), "The scope and goals of economic science. A Habermasian perspective », dans LAVOIE Don (ed.), Economics and Hermeneutics, Londres, Routledge. 